Musées, Patrimoine et Culture scientifiques et techniques

\title{
Formol et fixation : nouvelle donne, nouvelles approches...
}

André Clique, Jean-François Ponge, Yves Le Gal et Dominique Especel

\section{OpenEdition \\ Journals}

Édition électronique

URL : http://journals.openedition.org/ocim/695

DOI : $10.4000 /$ ocim.695

ISSN : 2108-646X

Éditeur

OCIM

Édition imprimée

Date de publication : 1 novembre 2007

Pagination : 23-29

ISSN : 0994-1908

Référence électronique

André Clique, Jean-François Ponge, Yves Le Gal et Dominique Especel, « Formol et fixation : nouvelle donne, nouvelles approches... », La Lettre de l'OCIM [En ligne], 114 | 2007, mis en ligne le 03 février 2011, consulté le 19 avril 2019. URL : http://journals.openedition.org/ocim/695 ; DOI : 10.4000/ ocim. 695 


\title{
Formol et fixation : nouvelle donne, nouvelles approches...
}

\author{
André Clique, Jean-François Ponge, Yves Le Gal et Dominique Especel *
}

\author{
Mise au point par les chercheurs du Muséum \\ national d'Histoire naturelle à la station de \\ Biologie marine de Concarneau, la technique \\ décrite ici consiste à fixer des poissons au \\ formaldéhyde sous hyperbarie. Élaboré avec \\ pour souci principal une exposition la plus \\ réduite possible du manipulateur aux vapeurs \\ de formol, le champ d'application de \\ ce nouveau procédé s'étend notamment \\ à la sauvegarde, au conditionnement \\ et au transport de certains spécimens \\ des collections des muséums.
}

La nouvelle réglementation européenne sur les produits cancérogènes, mutagènes et repro-toxiques (INRS 2006) impose, si un produit ne peut être remplacé par un substitut, de réduire les quantités manipulées et d'assurer au mieux la protection des

* André Clique est ingénieur d'études CNRS au Muséum national d'Histoire naturelle, UMR 5178,

station marine de Concarneau clique@mnhn.fr

Jean-François Ponge est professeur au Muséum national d'Histoire naturelle, CNRS UMR 7179 Yves Le Gal est directeur honoraire de la station marine de Concarneau

Dominique Especel est ingénieur Hygiène et Sécurité au Muséum national d'Histoire naturelle, service d'Hygiène et Sécurité travailleurs exposés. Cette problématique intéresse $a$ priori tous les muséums dans la mesure où ils sont concernés par la conservation, le stockage et la présentation d'animaux ou de pièces anatomiques. Cette logique peut aussi être poussée plus en avant : en effet la logistique de transport d'animaux préparés provenant de contrées parfois très lointaines est amenée à évoluer avec les nouvelles mesures concernant le transport aérien entre autres (produits potentiellement explosifs ou classés inflammables).

Nous avons travaillé en ce sens pour la fixation de poissons de taille petite à moyenne, cette méthode étant aussi applicable à des petits mammifères ou des pièces anatomiques diverses. Le principe de base retenu est celui d'une saturation des tissus sous pression afin d'assurer la fixation des échantillons à cœur. La désaturation s'effectue avec un protocole de type "paliers de décompression » par assimilation à ceux mis en pratique lors des remontées en plongée sous-marine. La neutralisation du formaldéhyde s'effectue en phase finale. Le conditionnement a aussi fait l'objet de notre attention ainsi que la possibilité de traitement et de conditionnement in situ des échantillons récoltés avant leur transport définitif vers les muséums concernés.

\section{Le principe de fixation de tissus au formaldéhyde sous pression (hyperbarie)}

Le formaldéhyde ou aldéhyde formique est un gaz commercialisé sous forme de solution dissoute aux environs de $40 \%$, appelée communément formol. Il est généralement utilisé à raison de quelques pourcentages de la solution mère dans l'eau. Le principe de fixation retenu est une saturation directe des tissus par le gaz formaldéhyde dissous mis sous pression. 
Du point de vue physiologique (modèle Haldane), on considère qu'il existe des groupes de tissus différents, allant de la chair à l'os appelés « compartiments » (Juvensen et Thomas, 1997). Cette classification est basée sur le fait que les tissus sont plus ou moins facilement en contact avec les gaz (cas des poumons par exemple) ou le sang circulant. Les tissus qui sont saturés en dernier sont donc les os et les graisses.

D'après les données des tables de plongée, qu'elles soient américaines (US Navy, 2005) ou françaises (FFESSM 2000), la saturation de tous les groupes de tissus est complète au bout de 7 périodes soit 720 minutes ou encore 12 heures. Dans le cas présent il convient de ne pas perdre de vue que nous avons affaire à des tissus morts, c'est-à-dire ni ventilés ni irrigués. Les valeurs à prendre en compte sont donc bien celles des deux derniers groupes de tissus (os et graisse), même si nous avons affaire à des tissus musculaires.

Au bout de ce séjour à 2 bars (soit l'équivalent d'une plongée de 12 heures à $-20 \mathrm{~m}$ ), les tissus sont donc saturés en formaldéhyde sur toute leur épaisseur. Commence alors le processus de dépressurisation par paliers (comme une remontée vers la surface dans une plongée classique longue). Des paliers de plusieurs heures à différentes pressions intermédiaires sont alors effectués jusqu'à désaturer l'ensemble des tissus. Les protocoles de paliers ont pour origine les tables de la Marine nationale dites MN90 (plongée prolongée à $-60 \mathrm{~m}$ ), soit une marge de sécurité d'un facteur 3 (FFESSM 2000). Ces paliers pris en compte à partir de $-20 \mathrm{~m}$ ont cependant été modifiés et adaptés à nos besoins.

\section{Comment déterminer les constantes de temps et de pression des paliers de désaturation?}

À l'origine nous souhaitions travailler directement en phase vapeur de formaldéhyde saturante et sous pression d'air. Le danger de l'expérimentation ainsi que le fait que le formaldéhyde se détruise en présence d'oxygène (Batista et Iwasita, 2006) nous en ont dissuadé. Cependant nous nous sommes servis d'un montage expérimental pour mettre au point les protocoles de paliers. Pour des raisons d'asepsie (les échantillons sont expérimentés sur plusieurs jours à température ambiante), nous avons opté pour le protocole suivant :

- les échantillons sont placés dans une enceinte dite « gaz »;

- l'enceinte dite « liquides » est remplie d'un peu de formol à $5 \%$. Une pompe à vide/compresseur, dépressurise l'enceinte liquide, et fait passer le formaldéhyde sous forme vapeur et l'envoie dans l'enceinte « gaz » en quelques minutes ;

- un dispositif de barbotage d'air dans un bain de sulfite est alors mis en place afin d'éliminer l'oxygène de l'air pour la séquence de compression ;

- la même pompe, en position compresseur cette fois, pressurise alors les échantillons à 2.0 bars pour environ 24 heures ;

- une vanne de micro débit permet ensuite de faire redescendre la pression par paliers (les vapeurs résiduelles sont piégées dans un filtre en sortie).

La présence d'une faible quantité de formol sous forme vapeur est insuffisante pour fixer les échantillons mais assure une stérilisation partielle des échantillons qui se décomposent alors plus lentement à température ambiante. Différents protocoles de paliers ont été testés sur diverses espèces. Pour la pression des paliers intermédiaires nous avons retenu les valeurs suivantes : 1.7 bar, 1.4 bar, 1.0 bar, 0.6 bar, 0.3 bar.

Il ne nous est pas actuellement possible, dans le cadre de cet article, de communiquer les détails des paliers ainsi que ceux des vitesses de transit d'un palier à l'autre, l'ensemble du procédé ayant fait l'objet d'un dépôt de brevet à l'INPI (institut national de la Propriété industrielle).

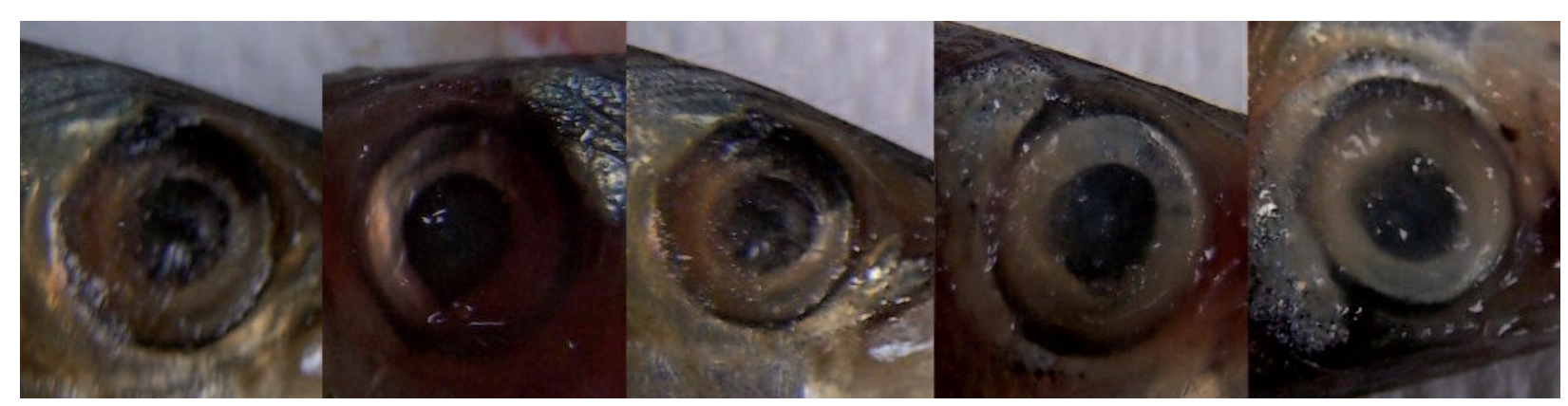

Absence de bulles dans l'humeur vitrée de l'œil des sardines (c) station de Biologie marine 


\section{Comment s'assurer de la bonne exécution des paliers et du respect du temps de passage d'un palier à l'autre ?}

Le test retenu pour s'assurer de la bonne exécution de la procédure de désaturation a été celui de la présence de bulles dans l'humeur vitrée de l'œil, en accord avec la médecine ophtalmique hyperbare (www.snof.org/maladies/diving.html).

Les espèces ayant été testées sont les suivantes : dorades, tacauds, chinchards, sardines. Chez les sardines fraîches, il n'a pas été observé de bulles dans les cavités oculaires sous loupe binoculaire. Par contre deux animaux avaient la peau abîmée. Il n'a pas été possible de savoir si cette dégradation était due au fait que les animaux avaient séjourné de nombreuses heures à température ambiante ou si ce phénomène était dû à un problème de désaturation, les sardines étant par nature fragiles à la manipulation. Pour les chinchards, tacauds ou dorades nous n'avons pas observé de lésions de surface ni de présence de bulles dans l'humeur vitrée.

\section{Nos expérimentations de fixation d'échantillons (poissons) sous hyperbarie}

Les différentes étapes de mise en fonctionnement sont les suivantes :

- le réservoir de transit est rempli de 10 l d'eau et un film d'huile (50 ml) est déposé en surface ;
- les échantillons sont disposés dans l'enceinte expérimentale :

- le robinet de purge du circuit d'air est ouvert, la vanne de vidange également. L'enceinte expérimentale se remplit par gravité. Vanne et robinet sont fermés ;

- mise sous pression à l'aide d'un compresseur (2 bars) ;

- fixation (24 ou 48 heures) à 2 bars ;

- paliers, pour passer d'une pression à l'autre la vanne de micro débit est ouverte. Le débit est du type bulle à bulle. Une fois la pression requise atteinte, la vanne est fermée en attente du palier suivant ;

- vidange après retour à la pression atmosphérique : la vanne de vidange est ouverte, environ 0.15 bars sont injectés dans l'enceinte. Le liquide et l'huile sont refoulés dans le réservoir de transit. La vanne trois voies isole le tout ;

- ouverture de l'enceinte expérimentale. Sortie des échantillons sous film d'huile, rinçage, mise sous éthanol.

S'il n'y a pas de nouveaux échantillons à traiter on peut neutraliser le formol par vidange dans la cuve de neutralisation contenant une solution aqueuse de bicarbonate ou de carbonate d'ammonium (Kawamata et Kodera, 2004).

Plusieurs essais ont été effectués en juillet 2007 sur des lots de 4 ou 5 maquereaux congelés ou non. Il n'a pas été rencontré de difficultés particulières. Les animaux semblent correctement fixés comparativement

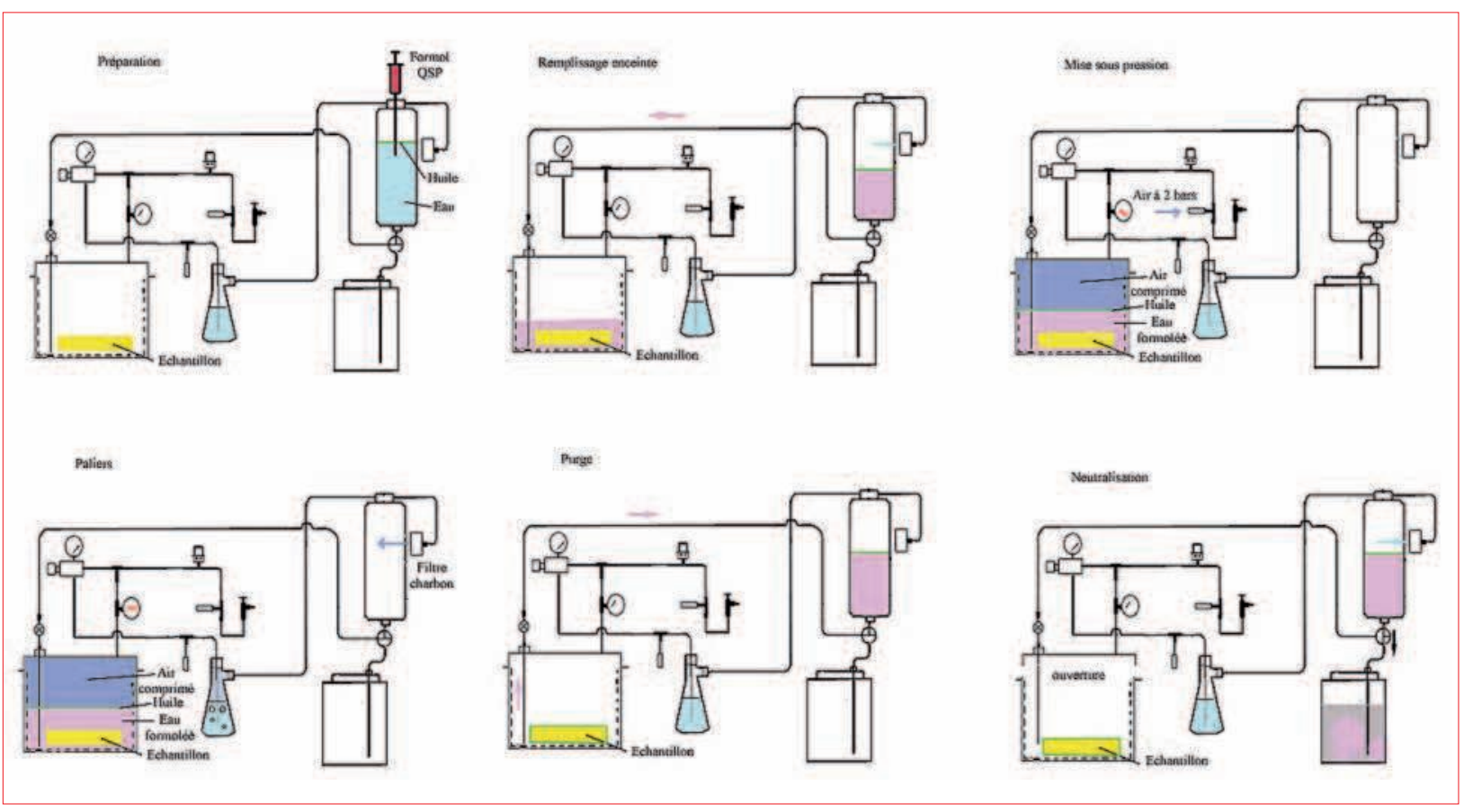

Résumé du processus de fixation (c) station de Biologie marine 
aux témoins traités de manière classique (séjour d'une semaine dans un bain à $5 \%$ ).

\section{Poursuivre la logique de prévention sécuritaire jusqu'au bout}

\section{Le rinçage}

Pour le rinçage des animaux après fixation et par souci d'économiser l'eau autant que celui de soustraire l'expérimentateur aux vapeurs résiduelles de formol, nous avons mis au point une machine à pulvérisation en vase clos. Cette machine permet également de formoler des petites quantités d'animaux à pression atmosphérique et de façon totalement étanche.

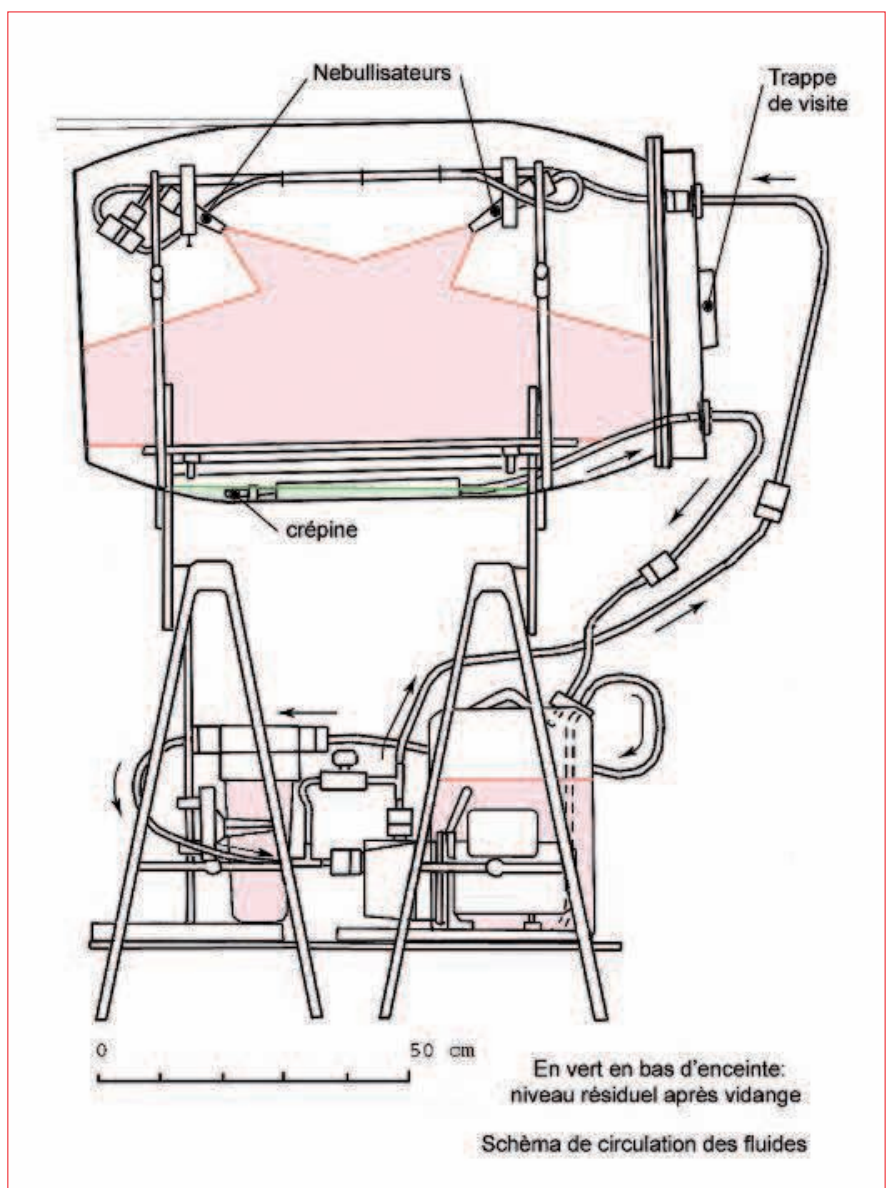

Machine à formoler et à rincer : en mode dit de "fixation ", la détente assure la vaporisation du formol en vase clos. (c) station de Biologie marine

La mise sous alcool

Bien que l'alcool ait des effets secondaires euphorisants, il n'en reste pas moins un toxique et doit être à ce titre l'objet d'attentions particulières. Pour les échantillons de taille raisonnable (maxi $30 \mathrm{~cm}$ ), nous avons développé une technique nouvelle dans le souci également d'exposer le moins possible l'expérimentateur aux vapeurs d'alcool. Nous fabriquons des poches plastique thermosoudées et stockons l'alcool à $-30^{\circ} \mathrm{C}$. L'échantillon fixé est disposé dans la poche, l'alcool à $-30^{\circ} \mathrm{C}$ est versé dessus. Le tout est scellé par thermosoudage et sans risques, la tension de vapeur de l'alcool étant très faible à cette température. L'autre intérêt de la méthode consiste en une économie substantielle d'alcool allant jusqu'à -30\% par rapport à un conditionnement en flûtes par exemple. Pour les animaux de taille plus conséquente, le stockage en touque reste en usage (éventuellement sous glycérol, des essais en ce sens sont en cours au Muséum national d'Histoire naturelle).

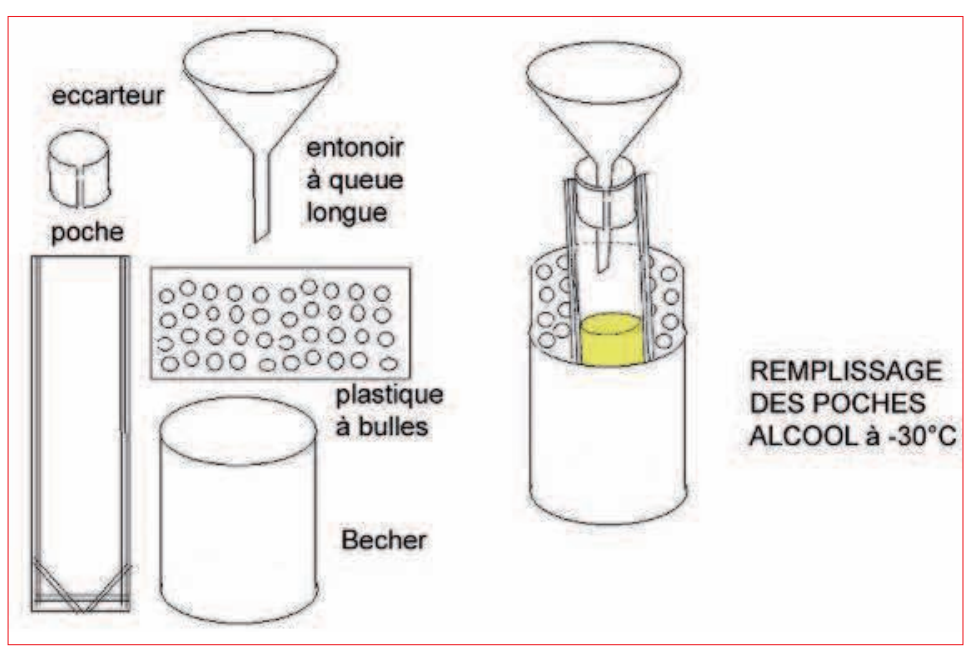

Conditionnement sous alcool à basse température (c) station de Biologie marine

\section{Réponses à quelques questions que l'on est en droit de se poser}

D’un aspect visuel, les écailles ont le même état de préservation qu'avec la technique de fixation classique par bain prolongé. Pour les poils et les plumes, aucune expérimentation en ce sens n'a été effectuée. La structure cellulaire et tissulaire ne semble pas affectée par le processus mais nous n'avons pas fait d'histologie particulière sur les échantillons. C'est l'expansion de ces bulles en cas de non-respect du protocole de paliers qui occasionne des lyses de cellules et provoque par ailleurs des embolies (chez les sujets vivants).

Le formol est connu pour dégrader l'ADN (ce qui n'est pas le cas de l'éthanol). Dans une cellule épithéliale, le formol réagit avec les protéines et les acides nucléiques au niveau des fonctions NH2 ou en 
faisant des pontages méthyléniques entre les protéines et les acides nucléiques (cross link) et en provoquant des cassures mono et double brins de l'ADN. Le souci de pérennité de l'échantillon ne se pose pas en termes réels dans notre cas, d'autant que l'éthanol est également un fixateur. Pour le conditionnement sous glycérol nous ne possédons pas de recul. Cet aspect du problème est traité par le laboratoire dit des "Pièces molles » au sein du Muséum national d'Histoire naturelle (département Milieux et Peuplements Aquatiques).

\section{Que conclure de cette nouvelle approche ?}

Ce procédé nouveau répond à nos trois objectifs initiaux :

- améliorer la sécurité de l'expérimentateur en supprimant l'exposition aux vapeurs de formol, en accord avec la nouvelle réglementation CMR de la Communauté Européenne (INRS 2006) ;

- diminuer le temps de contact des échantillons avec le formol ;

- obtenir un dispositif utilisable aussi bien au laboratoire que sur le terrain.

Le problème de l'exposition aux vapeurs est résolu : le système est totalement étanche dans sa phase hyperbare. Le rinçage des échantillons expose peu le manipulateur : la fine couche d'huile qui recouvre les échantillons à leur sortie d'enceinte diminue fortement l'exposition aux vapeurs de formol. Lélimination de cette pellicule d'huile s'effectue pendant le rinçage effectué en milieu clos sans intervention du manipulateur. Le formol est neutralisé par le bicarbonate d'ammonium. Le procédé (gravité) est tel que l'huile se retrouve en surface en fin de neutralisation. Les vapeurs éventuelles sont ainsi très limitées. Le bicarbonate d'ammonium nous semble la solution la plus adaptée à la neutralisation : nous avons testé également l'ammoniaque (technique hospitalière) qui neutralise le formaldéhyde en formant de l'hexamine (Frosin et al., 1980) et l'hypochlorite de sodium ou eau de Javel. Ces deux derniers produits imposent l'usage d'un masque mais le bicarbonate d'ammonium, seulement irritant pour la peau, ne requiert que des gants. De plus l'eau de Javel peut dans certaines conditions réagir avec le formol en donnant des produits explosifs (Walker, 1975) et du bischlorométhyléther, un carcinogène puissant (www.ch-aix.fr/pro/theme/anapath/prevrisques.html). Nous n'avons pas testé la destruction des aldéhydes par le permanganate de potassium (Picot et Grenouillet, 1992).
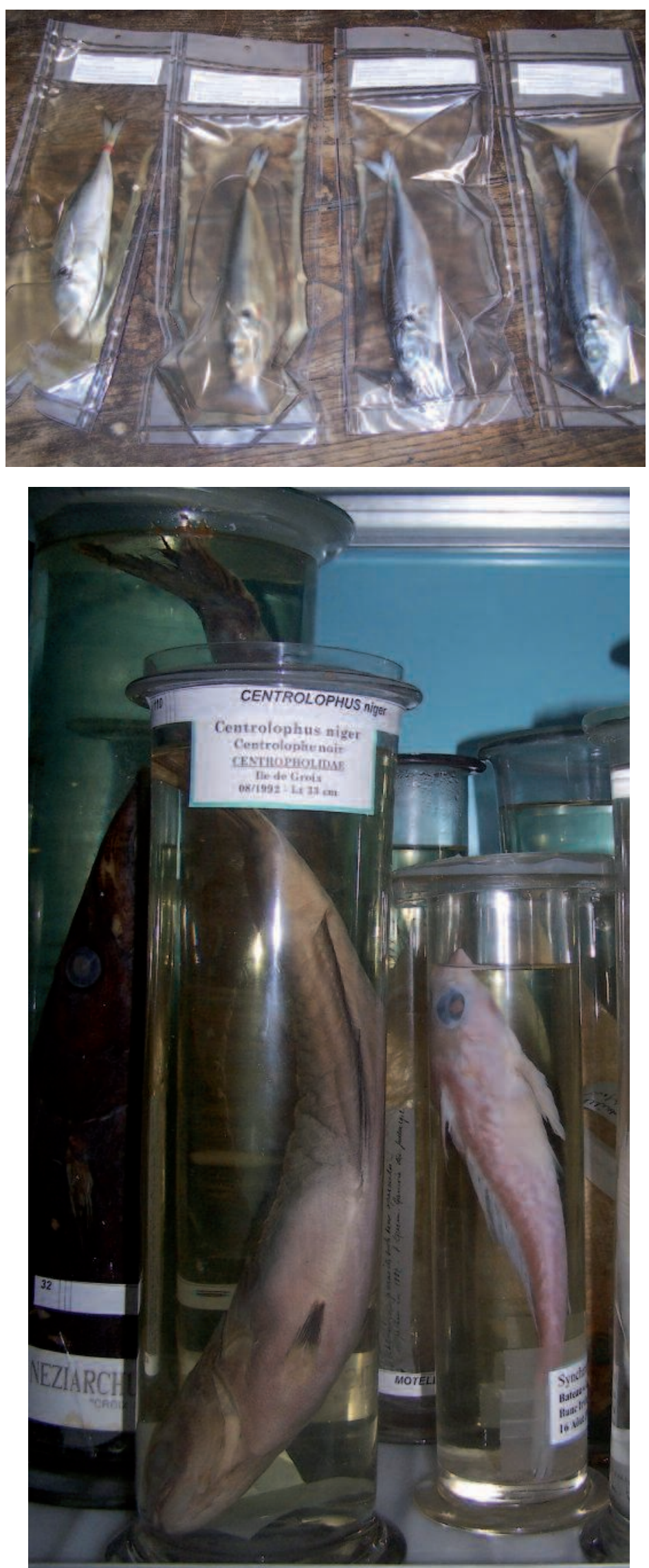

Échantillons de chinchards conditionnés en poches plastique et conditionnement classique en flûtes () station de Biologie marine 
La diminution du temps nécessaire pour la fixation des tissus fait également partie des objectifs d'origine. La saturation des tissus par hyperbarie y répond. Le temps de macération sous formol est sensiblement réduit par rapport à la méthode classique d'immersion. Par exemple des échantillons de grande taille nécessitant habituellement environ un mois de contact en bain de formol, auquel s'ajoute le temps de rinçage, peuvent être traités en 48-72 heures.

Le rinçage en vase clos est économique en eau : un rinçage habituel consomme environ 7200 litres d'eau $(5 \mathrm{l} / \mathrm{mn})$ contre 5 fois 10 litres pour notre méthode. Il faut également remarquer que dans l'enceinte, le volume d'air comprimé est faible par rapport à celui du mélange eau/formol (c'est aussi un critère de sécurité d'explosion sous pression). Cependant la présence de micro-fuites sur des périodes longues peut mettre en péril l'expérimentation si elle n'est pas contrôlée par un automatisme. Par précaution, nous enduisons nos joints de Loctite $5923{ }^{\circledR}$ dit « joint poisseux ».

La mise au point des paliers de décompression s'est avérée empirique en l'absence de données sur des tissus morts. On peut cependant considérer que le l'observation des bulles dans les yeux des animaux (www.snof.org/maladies/diving.html) reste un critère assez fiable de la réussite ou non de la procédure de décompression.

Toutes nos expérimentations on été réalisées en laboratoire. Ce n'est pas pour autant que la mise au point d'une méthode utilisable sur le terrain a été négligée (pour de petits mammifères exotiques et à la demande d'utilisateurs). Dans le laboratoire certaines facilités nous ont été accordées (compresseur électrique par exemple). Sur le terrain ont peut envisager une mise sous pression manuelle ou une alimentation en gaz sous pression à partir d'une bouteille de plongée par exemple. Tous nos paliers ont été réalisés de manière manuelle (immédiatement applicable sur le terrain). Rien m'empêche cependant d'envisager un pilotage des paliers par un automate et des sondes de pression (que ce soit en laboratoire avec alimentation sur secteur ou sur le terrain avec une alimentation par batterie). L'avantage d'un système piloté serait double : d'une part il serait possible de compenser automatiquement d'éventuelles micro-fuites, d'autre part l'expérimentateur ne serait plus assujetti à un planning fortement lié aux périodicités des opérations de décompression à effectuer.
Il est à noter qu'il serait inconvenant de notre part de ne pas prendre en compte la mise sur le marché de produits de substitution (fixateurs ou conservateurs). Cependant, en l'état actuel de nos connaissances, il semble qu'ils ne soient susceptibles d'être appliqués qu'à des coupes histologiques ou des échantillons de taille extrêmement réduite, ce qui les exclut de notre problématique actuelle. En ce qui concerne les produits de substitution, nos réflexions sont les suivantes (basées sur une analyse de M.-C. Yéprémian). Pour les différents types de substituants, le substituant idéal serait un aldéhyde mais moins toxique et moins volatil que le formaldéhyde. La molécule se rapprochant le plus de cet idéal serait le glyoxal. Selon le fabricant du PREFER ${ }^{\circledR}$ (formulation avec du glyoxal) la tension de vapeur de cette molécule est très faible, ce qui est vrai par rapport au formaldéhyde mais faux dans l'absolu, le glyoxal étant aussi une molécule volatile. Le glyoxal est plus de 15 fois moins volatil que le formaldéhyde, l'équivalent américain de la valeur moyenne d'exposition (8 heures, VME) étant supérieur de 12 fois à celui du formaldéhyde. Il est classé malheureusement Mutagène 3 par l'INRS, mais on n'a aucune information sur son action sur la cancerogénèse ! Enfin, en se basant sur le prix au litre du PREFER ${ }^{\circledR}$, on peut envisager une augmentation par un facteur 5 du prix du litre de conservateur (comparaison avec une solution de formaldéhyde à $10 \%$ $(\mathrm{v} / \mathrm{v})$, catalogue Aldrich 2007). En conclusion, le formaldehyde est en fait une molécule à usage multiple (conservation, fixation histo-pathologique, action biocide) d'où la possible difficulté de trouver un substituant combinant l'ensemble de ses qualités.

Le domaine d'application des solutions que nous proposons couvre, au moins partiellement, l'aspect de sauvegarde (formol, alcool) et de conditionnements (glycérol, alcool) des collections. Il nous semble que diverses institutions pourraient être concernées (musées des facultés de Médecine et des écoles vétérinaires, muséums d'Histoire naturelle) ainsi que leurs secteurs de recherche. Le conditionnement sous glycérol nous semble d'autre part une piste intéressante à deux titres dans une optique sécuritaire et hygiénique : le transport et la présentation au public.

Les auteurs tiennent à remercier Elena Luchetti et S. Iglésias de la station de Biologie marine de Concarneau pour leur aide technique, le comité d'Hygiène et de Sécurité du Muséum national 
d'Histoire naturelle pour son soutien et ses encouragements ainsi que Claude Yéprémian, responsable de l'Algothèque (souches non toxiques) au Muséum national d'Histoire naturelle.

\section{Bibliographie}

Batista, E.-A. et Iwasita, T. Adsorbed intermediates of formaldehyde oxidation and their role in the reaction mechanism, Langmuir, vol. 22, 2006, pp. 7912-7916.

FFEFFSM Table Marine nationale 1990 MN90, version du 12/1/2000. http://plongee.asparis7.com/pdf/mn90.pdf

Frosin, V.-N., Tsibikov, V.-B., Izvekova, G.-I., Rabin'kii, B.-Y., Pakhomov, S.-V., Ramkova, N.-V., Alekseeva, M.-I., Kareev, N.-V., Likhtman, T.-V., Ryabov, P.-I. et Komarkova, N.-I. Selection of a mode for formaldehydegas sterilization, Biomedical Engineering, vol. 14, 1980, pp. 178-181.
INRS, Produits chimiques cancérogènes, mutagènes, toxiques pour la reproduction. Classification réglementaire. Aide-mémoire Technique ED 976, 2006.

Juvenspan, H. et Thomas, C. Plonger aux mélanges. Paris : Eugen Ulmer, 1997, $186 \mathrm{p}$.

Kawamata, S. et Kodera, H. Reduction of formaldehyde concentrations in the air and cadaveric tissues by ammonium carbonate, Anatomical Science International, vol. 79, 2004, pp. 152-157.

Picot, P. et Grenouillet, P. La sécurité en laboratoire de chimie et de biochimie. Paris : Lavoisier, 1992, 424 p.

US Navy Diving Manual, Revision 5, 2005. www.supsalv.org/manuals/ diveman5/divManual5.htm

Walker, J.-F. Formaldehyde. New-York : Krieger, Huntington, 1975 